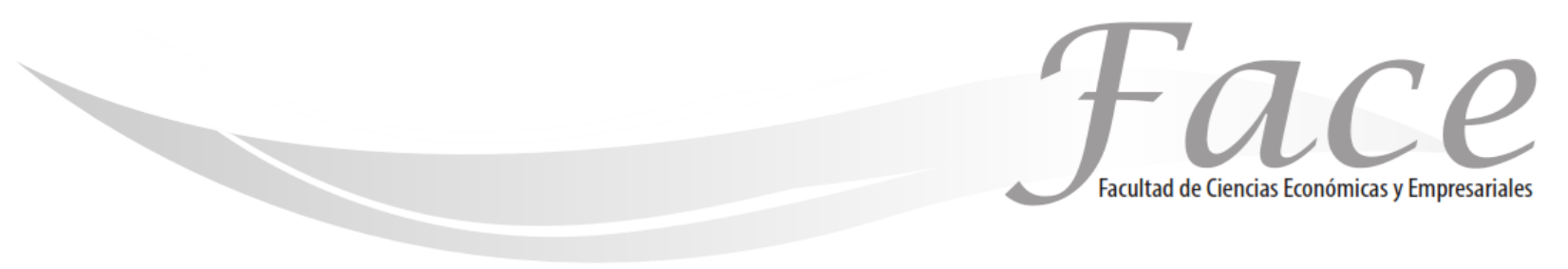

ISSN 1794-9920

Volumen $15-2$

Año 2015

Págs. $107-116$

\title{
APLICACIÓN DEL MODELO HEURISTICO SIGNIFICATIVO EN LA INTERPRETACIÓN DE LA CULTURA AMBIENTAL*
}

Fecha de Recepción: 29 de Septiembre 2015

Fecha de Aprobación: 15 de Diciembre 2015

\author{
Doris Vanegas Vanegas** \\ Jacipt Alexander Ramón Valencia \\ Jarol Derley Valencia
}

\begin{abstract}
Fecha de Aprobacion: 15 de Dlciembre 2015
\section{Resumen:}

Este estudio se propuso organizar los diversos aspectos de la cultura que suelen estar dispersos en diversas formas de alusión cotidiana y aún disciplinar, pero que no encuentran un referente discursivo, a pesar de la naturalidad con la que se expresan sobre lo cultural. Para ello, se basó en el modelo heurístico significativo y la heurística como método que a su vez se fundamenta en la observación, la categorización, la jerarquización, la representación y la justificación lógica. Con su aplicación, se logró representar interactivamente las relaciones que tienen lugar entre la Cultura, la Realidad, la Investigación, la Socialización y la Educación, principalmente, pero que pueden tener incidencia en la generación de una Cultura ambiental; se precisaron categorías relevantes a la hora de abordar temas culturales; se puntualizaron variables de intervención que posibilitan un diagnóstico, intervención y valoración de una problemática socio-cultural. Con la precisión de los aspectos culturales abordados, se evidenció la utilidad del método heurístico y de su replicabilidad en otros estudios. La gran importancia que está adquiriendo la Cultura para solucionar problemas sociales en relación a la educación, la ciudadanía, la paz y el comportamiento ambiental, hace necesaria la generación de un estado del arte que precise categorías, unidad de estudio y posibles variables de intervención.
\end{abstract}

Palabras Claves: Cultura, imaginario, acontecimiento, heurística, ambiental.

\footnotetext{
* Artículo resultado del Grupo de Investigación en Lenguaje, Educación y Cultura - IN LINGUA Programa de Lengua Castellana y Comunicación de la Universidad de Pamplona, y el Grupo de Investigaciones Ambientales Aguas, Aire y Suelo -GIAAS, programa de Ingeniería Ambiental Universidad de Pamplona - Colombia.

** Docente investigador, Grupo de Investigación en Lenguaje, Educación y Cultura - IN LINGUA Programa de Lengua Castellana y Comunicación de la Universidad de Pamplona. Contacto: dvanegas@unipamplona.edu.co

*** Docente Investigador de la Universidad de Pamplona, Grupo de Investigaciones Grupo de Investigaciones Ambientales Aguas, Aire y Suelo -GIAAS, programa de Ingeniería Ambiental Universidad de Pamplona. Contacto: jacipt@unipamplona.edu.co

**** Docente Investigador de la Universidad de Pamplona, Grupo de Investigaciones Grupo de Investigaciones Ambientales Aguas, Aire y Suelo -GIAAS, programa de Ingeniería Ambiental Universidad de Pamplona. Contacto: jramon002@ikasle.ehu.es
} 


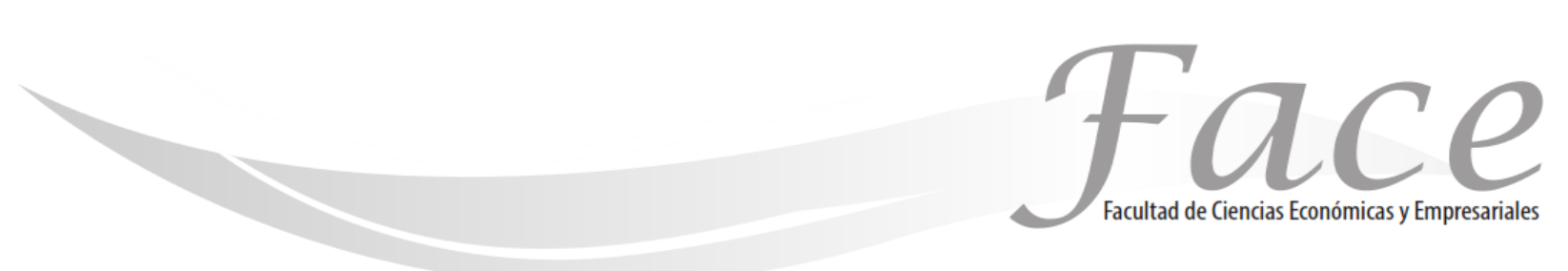

\title{
APPLICATION OF THE HEURISTIC SIGNIFICANT MODEL IN THE INTERPRETATION OF ENVIRONMENTAL CULTURE
}

\begin{abstract}
:
The purpose of this study was to organize various cultural aspects that are scattered in everyday life and in different disciplinary aspects, but are not found in a reference discourse, despite the ease in which they are expressed. The study was based on the heuristic significant model which is itself founded on observation, categorization, hierarchy, representation and logical justification. With its implementation, it was possible to interactively represent the relationship between Culture, Reality, Research, Socialization and Education, of which may have an impact on the generation of an environmental culture; relevant categories to address cultural topics were specified; interventional variables that enable diagnosis were identified, intervention and assessment of socio-cultural issues were emphasized. With the identification of the cultural aspects, the usefulness of the heuristic method and its replicability in other studies was demonstrated. The growing importance that culture is acquiring to solve social problems related to education, citizenship, peace and environmental behavior, requires the generation of a state of the art that specifies categories, study units and possible variables of intervention.
\end{abstract}

Keywords: culture, imaginary, event, heuristic, environmental.

\section{APLICAÇÃO DO MODELO HEURÍSTICO SIGNIFICATIVO NA INTERPRETAÇÃO DA CULTURA AMBIENTAL}

\begin{abstract}
Resumo:
Este estudo propõe-se a organizar os vários aspectos da cultura que estão dispersos em várias formas de referência quotidiana e disciplinar, mas que não possui um discurso referente, apesar da naturalidade com que são expressas no cultural. 0 estudo baseou-se no modelo heurístico significativo e o método heurístico, que por sua vez esta fundamentada na observação, categorização, hierarquia, representação e a justificação lógica. Com sua aplicação, foi possível representar interativamente as relações que ocorrem entre a cultura, realidade, investigação, socialização e educação, principalmente, e que podem ter um papel na geração de uma cultura ambiental; categorias relevantes foram definidas para lidar com as questões culturais, foram especificadas as variáveis de intervenção que permitem um diagnóstico, intervenção e avaliação de um problema sociocultural. Com a identificação dos aspectos culturais, comprovou-se a utilidade do método heurístico e sua replicabilidade em outros estudos. A grande importância que está obtendo a cultura para resolver os problemas sociais em relação à educação, cidadania, paz e o comportamento ambiental, requer a geração de um estado de arte com categorias definidas, unidades de estudo, e possíveis variáveis de intervenção.
\end{abstract}

Palavras-Chave: Cultura, imaginário, evento, heurístico, ambiental. 


\section{INTRODUCCIÓN:}

Abordar epistemológicamente un objeto de estudio tan amplio y reconocido como lo es la Cultura, se convierte en un gran reto por resolver. Realidad cognoscible que es susceptible de ser abordada desde la heurística para reconocer puntualmente sus características, elementos, evolución, ámbitos, contexto y demás aspectos que evidencian su complejidad.

Incursionar en la Cultura supone contar con unos principios coherentes que, en este caso, se identifican con el enfoque sociocultural (Martínez, 1999), la heurística (López, 2005). y la complejidad (Morin, 1994). Principios como la motivación, la creatividad, la textualización, la contextualización, la interacción y la resonancia, son coherentes con cada una de estas miradas.

La motivación ha sido el móvil para la mayoría de las acciones humanas; ya sea suscitado por la necesidad 0 por la curiosidad. El ser humano ha sido creativo por naturaleza y, resultado de ello, tenemos a la Cultura. De ella, van quedando vestigios reconocidos como formas de textualidad que se conciben dentro de una contextualización que permite ubicar a cada hecho cultural en un momento histórico y un espacio geográfico; sin embargo, este principio trasciende hacia lo psicológico, lo verbal, lo experiencial y a lo interpersonal, lo regulativo, lo experiencial y lo instructivo en términos de Berstein, parafraseado por Halliday (2013). La interacción, aunque tiene que ver con lo interpersonal, es principio vital para el desarrollo de lo cultural que se evidencia a su vez con otro principio como la resonancia, impacto que va generándose a medida que una cultura se expande.

Este efecto dominó, entroniza un cambio continuo, dinámico e interactivo que propicia una gran influencia de la Cultura y sus microculturas sobre el ser humano actual, sobre sus decisiones y forma de vida; razón para emprender en estos tiempos del siglo XXI un estudio acucioso sobre ella y precisar elementos cruciales para su comprensión.

Si se toman coordenadas espacio-temporales, vemos que el contexto en el que se manifiesta es tan amplio que abarca todo ámbito de la actividad humana, todo un radio de acción sobre el medio, sobre el hábitat. De ahí que no sea sencillo precisar sus categorías ni sus fronteras microculturales. Una posible representación del fenómeno de culturización y sus categorías inmediatas es la siguiente: Ver figura 1.
Figura $\mathrm{N}^{\circ} 1$. Fenómeno de Culturización

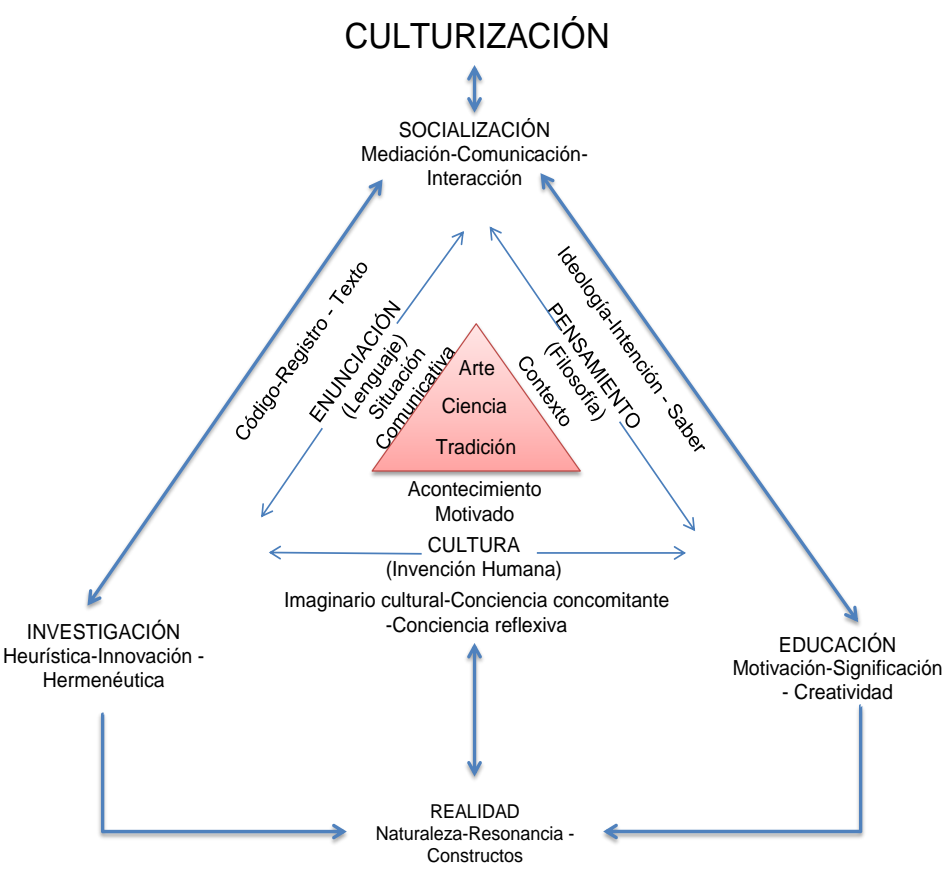

Fuente: Elaboración propia.

En esta representación, tienen lugar otros macrofenómenos culturales como la investigación, la socialización y la educación que actúan interactivamente valiéndose de la enunciación, el pensamiento y el estado cultural de las instancias que activan el proceso de culturización en una realidad regida por sus propias leyes y que inciden en dicho proceso.

A continuación se presenta una síntesis del Modelo heurístico significativo desde el cual es posible incursionar en objetos de estudio. (Vanegas y López, 2005). 
MODELO HEURISTICO-SIGNIFICAT NO

Un camino hacia la conciencia reflexiva y a la contribución cultural

15 Estrategias de comun icación y concientización reflexiva. Con tepución al código cultural.

14 Investigativamente, un objeto puede verse como problerpa Vanálisis con árbol de problemas y objetivos). Matriz de marco lógico: precisión de población y muestra; selección y ap licación de método(s), in strumentos, observaqión y ragistro; codificación de datos y tabulación; resultados estadísticos, seg n caso, y cualitativos; comportamiento de variables en observación-intononniónem etacogniciołp; resultados y discusión; conclusiones; revisjón-té ímpactos; declaraciones de conocimiento y de valor; estrateg ias de comunucación de avances y resultados; y, publicación-diśminación.

13 Trasciende discursiyamente a la descripción, la explicación, la argumentación y la proposición.

12 Compren sión yerica, metodológica, teleológica (pensamiento formal: +concepto s): Reconocimiento teórico-metodológico-teleológico del objeto de estudio, de relaciones, interaccighes, contrastes...(Representación del constructo teórico acerca del objeto/de estudig.

11 Clarigad en definiciones, conceptos y su representación cognitiva (pensamiento con (eptual:tproposiciones y formal: +conceptos): mapa conceptual/meytefacto $/ \mathrm{v}$ herrística/...; para ello, planteamiento de preguntas focales desde/lo teórico (ofmportamiento, funcionamiento, acontegimientas.prosesgs, fenómenos...); desde lo téleológico (filosofías, ideologías enfoques...); y, desde lo metodológico (procedimientos, métodos....

10 Lectura textual-referenciaycomprensiva) -contextual-i ferencral (interpretativa)intertextual-crítica (argumfntativa) transtextual-interactiva (fropositiva), estrategias de lectura y registro ara comprensión-interpretación- Jumentación-proposición: Reconocimiento de macrdestructuras culturales y disciplin ares, de pensamiento autores. macroestructuras/aspect ds/variables-prin cipios y criterios-normas/reglas, métodos/procedimien tos-pplicación, antecedentes-estado de arte.

9 Idea del objeto de studio-afirmaciones, negaciones, preguntas (pensamiento proposicional: thociones)

8 Plan de indagación categ drís básicas- pensamiento nocional.

7 Presaberes e imaginario đultural (representación previa del objeto de estudio: imágenes, actitudes, nociones, conc pptos, proced imien tos, métodos...).

6 Planteamien to de pregunt 1 s focales desde lo nocional a lo formal.

5 Selección de categońá-uso $v$ heurístico-significativa de pensamiento nocional, proposicional, conceptua y formal.

4 Selección de herramienta cognitiva según naturaleza del objeto: $v$ heurística-mentefactosistema complejo...

3 Selección de objeto de es udio.

2 Sujeto indagador-ser situado en mundo cognoscible-con función heurística-cognitivacomun icativa-cultural-ling iística.

1 Mundo cognoscible: ser (propiedades)-relaciones-acontecimientos-situación espacialtemporal.

\author{
FINALIDAD: INVESTIGACIÓN- SOCIALIZACIÓN - EDUCACIÓN
}

\section{MARCO TEÓRICO:}

Definir los aspectos más representativos de la Cultura como fenómeno global, así como su origen y evolución en el caso de Occidente, se torna en una aventura muy interesante. Para esta incursión, es necesario revisar las concepciones que se pueden titular "emblemáticas" sobre la Cultura; cada disciplina a su manera ha sentido el interés de conocer su origen y evolución.

Por consiguiente, la Cultura puede considerarse como la gran suma de todos los avances en cada una de las disciplinas, de las manifestaciones artísticas, de la tradición y de la cotidianidad. Veamos cómo ha sido el acercamiento a la Cultura a lo largo del tiempo y desde qué perspectiva.
2.1 Registro de La Concepción Ideológica de la Cultura a Través del Tiempo

"Cultivación del alma". Cicerón, 45 a. C. en Tusculanae Disputationes (Nuevo Idealismo/Estoicismo).

Parcela cultivada. S. XIII. (Concepción desde el estado de las cosas).

Cultivo de la tierra o el cuidado del ganado. S. XVI. (Concepción como actividad humana).

"Superación del barbarismo original". Pufendorf, s. XVII. (Concepción de nuevo estado humano posterior a un proceso).

Cultivo del espíritu. S. XVIII. (Formación de la mente, estado de la mente). 
Doris Vanegas Vanegas - Jacipt Alexander Ramón Valencia - Jarol Derley Valencia

"El motor del cambio histórico era el cambio de los medios de producción". Kames, 1761 en Historical Law Tracts. (Teoría de los estadios de desarrollo de la sociedad).

Civilización semejante a Cultura. S. XIX. (Refinamiento de las costumbres).

"Totalidad compleja". Tylor, 1871 en Primitive Culture. (Descriptivismo).

"Es uno de los determinantes fundamentales del desarrollo individual. Aporta la mayoría del contenido del pensamiento y las herramientas de adaptación intelectual". Vygotsky, 1934 en Pensamiento y lenguaje. (Mirada estructuralista semiológico-social).

"Sistema de signos producidos por la actividad simbólica de la mente humana". Lévi-Strauss, 1958 en La antropología estructural. (Estructuralismo).

No desarrolla directamente el concepto de Cultura pero sí alude a las formas ideológicas en que los hombres "devienen conscientes": tratando el proceso del devenir consciente como un proceso definido, con su propia lógica, mecanismos y efectos, que no ha de ser condensado 0 fundido con otras prácticas sociales. (Marx, 1965). (Determinismo socio-económico).

Se relaciona con el término hegemonía, un proceso por medio del cual un grupo dominante termina imponiéndose sobre otro. Gramsci, 1965. (Determinismo histórico-social).

"Todo aquello con lo que el hombre afina y desarrolla sus innumerables cualidades espirituales y corporales" Concilio Vaticano II, 1965. (Mirada valorativa de lo cultural).

El ámbito de la ideología (el principal componente de la cultura) formada de sistemas de representaciones, imágenes y conceptos, "estructuras como se le imponen a la gran mayoría de los hombres. Son objetos culturales percibidos-aceptados-sufridos y actúan funcionalmente sobre los hombres mediante un proceso que se les escapa". Las ideologías son, por tanto, la esfera de lo vivido; la esfera de lo experimentado y no la del "pensamiento". Althusser, 1965. (Sobredeterminismo socio-estructural).

"Organización jerárquica de valores accesible a todo el mundo pero al mismo tiempo es un mecanismo de selección y exclusión". Foucault, 1981-1982 en Cursos del College de France . (Mirada crítico-social).

"Da al hombre la capacidad de reflexionar sobre sí mismo". UNESCO, 1982 en Declaración de México. (Consideración como campo de acción hacia el desarrollo).

"Es el producto de las relaciones históricas entre un grupo humano y su medio ambiente". White y Steward, 1992 en
La energía y la evolución de la cultura. (Consideración neoevolucionista)

La cultura debe ser considerada como tejidos de significado dentro de los cuales los humanos deben vivir (...), los humanos están modelados excesivamente por su cultura y no existen características humanas transculturales unificadoras. (Geertz, 1987).

"Información transmitida por aprendizaje social". Mosterín (1993) en Filosofía de la Cultura (Memes, unidades de información, concepción científica de la Cultura).

En la mirada de la complejidad, parte de la crítica y del rechazo de la Cultura mutilada y mutiladora organizada en disciplinas que tantos efectos negativos lanza a la práctica de cualquier actividad. (Morin, 1994). (Desde el pensamiento complejo).

En un mundo global todas las culturas son igualmente válidas y tienen que aportar al concierto de la cultura global. (Magendzo, 2008). Hall (1981). (Mirada desde la globalización).

"Se transforma en el recurso fundamental para el desarrollo de comunidad y sociedades creativas, que permite la socialización del saber y el cultivo de las actitudes solidarias necesarias para alcanzar el desarrollo sostenible". (Barbero, 2011). (Mirada de la sostenibilidad ambiental y el desarrollo local a partir de la intervención cultural).

\subsection{Prolegómenos Para Un Estudio De La Cultura}

\subsubsection{Componentes $Y$ Rasgos Tangibles $E$ Intangibles De La Cultura}

Dentro de lo tangible, o evidente, se relaciona lo concerniente a prácticas lingüístico-discursivas, económicas, artísticas, científicas, rasgos materiales, útiles e instrumentos, uso de recursos, conocimientos, categorizaciones, textos como producto humano, modas, costumbres, tradiciones, hábitos permanentes de comportamiento, actividades cotidianas, programas, manejo de contextos, profesionalización de las actividades, texto lingüístico (López y Vanegas, 2005), discurso oral, modos de vida, rasgos físicos intervenidos, exteriorizaciones ideológicas y de imaginario cultural, códigos culturales (Carvajal, 1996), reglas de conducta, discursos de legitimación, prácticas de entronización y desentronización, exteriorizaciones psicológicas (autoestima, carácter, temperamento, alteridad, afectividad, vocación, prospectiva, emoción, ternura, ocio,...), protocolos de comportamiento, relaciones y prácticas de poder, derechos y deberes, prácticas de salubridad, prácticas de sostenibilidad ambiental, prácticas ciudadanas/rurales, prácticas bélicas/pacifistas, prácticas 
productivas, exteriorizaciones religiosas, prácticas de calidad de vida, prácticas de trascendencia y ritos funerarios, prácticas de desarrollo integral, prácticas de sexualidad, creación de contextos, exteriorización de decisiones, prácticas lúdicas, exteriorizaciones estéticas, exteriorización del pensamiento estratégico, exteriorización de la interacción, exteriorización de lo moral, exteriorización de criterios, objetos valorados por clase social dominante y dominada, hegemonía, procedimientos, conceptos, expresión, emoción, símbolos, código, registro, constructos, competencias (López, Vanegas y López, 2007), disciplinas, investigación sistematizada, prácticas comunicativas, constructo educativo, tecnología, estilos, cambios, efectos, experiencias y vivencias registradas, historia, exteriorizaciones filosóficas, manejo del color, manejo del sonido, manejo de las formas, manejo de las perspectiva, manejo de la luz, exteriorización de la creatividad, entre otros; y, dentro de lo intangible, o no evidente a simple vista, tenemos ideología, imaginario cultural, filosofía, características intelectuales, rasgos espirituales y afectivos, saberes, creencias, mitos, supersticiones, aspiraciones, principios, sistema de valores, criterios, moral, estética, capacidades, propósitos, estrategias, creatividad, interpretaciones, decisiones, conciencia concomitante, conciencia reflexiva, conciencia contextual, autoestima, carácter, temperamento, libre albedrío, alteridad, comunión, vocación, prospectividad, emoción, ternura, ocio, interacciones, saber disciplinar, saber investigativo, saber comunicativo, saber educativo, entre otros. De las anteriores categorías resaltan algunos conceptos o elementos que tienen gran relevancia al realizar consideraciones culturales:

Acontecimiento: Unidad de estudio de la Cultura. Se observa y analiza desde la complejidad. Contempla causas y efectos, participantes, relaciones, procesos y productos implicados.

Imaginario cultural: nociones, proposiciones, conceptos y teorías sobre la realidad como registro mental desde el cual se concibe, se interpreta, se argumenta y se propone.

Ideología: visión de mundo dependiente de una construcción socio-histórica (Torres, 1996). susceptible de cambio (Bajtin, 2011); perspectiva sobre las cosas, acontecimientos y comportamientos.

Comportamiento: exteriorización de la actitud, la concepción y el saber hacer.

Conciencia concomitante: conformidad y concordancia con lo establecido.

Conciencia reflexiva: interés en hallar inconformidades que permitan mejorar las condiciones existentes; pensamiento crítico y prospectivo responsable del cambio y del desarrollo.
Entronización: ideario y prácticas que permiten dar lugar preponderante a un personaje o a un acontecer ya sea momentáneo 0 permanente. Se entronizan leyes, derechos, fiestas, hábitos, procedimientos...

Desentronización: ideario y prácticas que permiten quitar el lugar preponderante a un personaje 0 a un acontecer ya sea momentáneo o permanente. Se desentronizan leyes, hábitos, procedimientos...

Tradición: es el conjunto de ideas, prácticas y elementos que han logrado ser entronizadas a lo largo del tiempo.

Regulación: mecanismo de mejoramiento a partir de la conciencia reflexiva.

Control: requerimiento de uso racional de ideas, prácticas y manejo de recursos.

Euforia: desenfreno o desbordamiento producto de la afición a una actividad, a un personaje 0 a unos elementos llamativos. Racionalizada, corresponde a la motivación, interés e inclinación hacia algo.

Bipolaridad: cambio repentino oscilante entre opuestos.

Ámbitos: esferas o campos de acción.

Código elaborado - científico: antecedentes y el estado del arte de una disciplina o profesión.

Lenguaje de primer nivel: lenguaje del ámbito cotidiano.

Lenguaje de segundo nivel: lenguaje del ámbito disciplinar o científico.

Conservación: ideal o propósito en el ámbito natural y cultural que impulsa a un uso racional de la ciencia y la tecnología.

Sostenibilidad: ideal o propósito en el ámbito natural y cultural que impulsa a un desarrollo racional social, científico y tecnológico.

Características de la Cultura: Dentro de sus rasgos tenemos que es un producto humano, un tejido social y un constructo del imaginario del mundo y la interacción y acción ejercida sobre él. Clasificación de la Cultura: según su extensión, particular/global; según su desarrollo, primitiva/civilizada; según su caracterización, sensista (desde lo sensitivo)/racional/ideal (racional y sensista); y, según su orientación, tradicional/contemporánea/futurista.

\section{METODOLOGÍA:}

3.1 Descripción y explicación. Se valen de la observación, el registro en diario de campo o cuaderno de trabajo, la entrevista, el registro fotográfico y otras evidencias para 
Doris Vanegas Vanegas - Jacipt Alexander Ramón Valencia - Jarol Derley Valencia

conformar el documental etnográfico, el reconocimiento de variables de observación con potencialidad de intervención para entronización de prácticas culturales en sociedades aspirantes a cambios, reflexivas y participantes, el registro del comportamiento de las variables, la categorización y clasificación, la sistematización, el inventario, el análisis, la síntesis, la interpretación, el catálogo y la comparación principalmente. prosperidad, del desarrollo integral urbano, entre otras. Se requiere de un talento humano comprometido y capacitado que ponga al servicio social y cultural su experiencia investigativa y su saber disciplinar. Esta forma de trabajo es complementaria a la observación, la explicación, la comprobación y otras formas de indagación.

Tabla $N^{\circ} 1$.

Heurística de la Cultura

\begin{tabular}{|c|c|c|c|c|c|}
\hline \multicolumn{6}{|c|}{ Cultura } \\
\hline \multirow{2}{*}{\multicolumn{6}{|c|}{$\begin{array}{l}\text { Leyes: complejidad, dialéctica bipolar, cambio, efecto dominó, fractalidad } \\
\text { Acontecimiento como unidad de estudio: Entronización, desentronización }\end{array}$}} \\
\hline & & & & & \\
\hline Principios: Motiv & \multicolumn{4}{|c|}{$\begin{array}{c}\text { Acontecimiento como unidad de estudio: Entronización, desentronización } \\
\text { Principios: Motivación, Creatividad, Textualidad, Contextualización, Interacción, Resonancia }\end{array}$} & \\
\hline \multicolumn{6}{|c|}{ Productos culturales humanos } \\
\hline $\begin{array}{l}\text { Tipos de } \\
\text { pensamiento } \\
\text { categorial, concreto } \\
\text { y abstracto, } \\
\text { Imaginario, } \\
\text { Lenguaje primer } \\
\text { nivel, } \\
\text { Lenguaje segundo } \\
\text { nivel, } \\
\text { Conciencia } \\
\text { reflexiva } \\
\text { Preguntas } \\
\text { Sociocrítica }\end{array}$ & $\begin{array}{l}\text { Uso y generación } \\
\text { de simbolos } \\
\text { (representa-ciones) } \\
\text { Convenciona- } \\
\text { lización de } \\
\text { actividades, } \\
\text { rituales, } \\
\text { hábitos } \\
\text { Conciencia } \\
\text { concomitante } \\
\text { Lo intangible }\end{array}$ & $\begin{array}{l}\text { Uso, transformación de } \\
\text { materialesy } \\
\text { aprovechamiento de } \\
\text { recursos naturales, } \\
\text { métodos, técnicas, } \\
\text { procedimientos } \\
\text { instrumentos }\end{array}$ & $\begin{array}{l}\text { Indagación, } \\
\text { Heuristica, Invención } \\
\text { de artefactosy } \\
\text { nuevas realidades, } \\
\text { Saber, competencia, } \\
\text { Hipótesis, } \\
\text { Variables, } \\
\text { Indicadores, } \\
\text { Protocolos } \\
\text { Lo tangible }\end{array}$ & $\begin{array}{l}\text { Creación de } \\
\text { constructos y } \\
\text { límites, Brecha, } \\
\text { Sectores, } \\
\text { Gremios, } \\
\text { Escuela, } \\
\text { Región de } \\
\text { conocimiento } \\
\text { Códigos } \\
\text { elaboradoso } \\
\text { científicos }\end{array}$ & $\begin{array}{l}\text { Generación de } \\
\text { fuerzas bélicas, } \\
\text { controly poder, } \\
\text { Generación de } \\
\text { fuerzas antibélicas, } \\
\text { Ideología }\end{array}$ \\
\hline $\begin{array}{l}\text { Lengua apoyada en } \\
\text { categorias, } \\
\text { Enunciación, } \\
\text { codificación, } \\
\text { decodificación, } \\
\text { Significación, } \\
\text { interpretación, } \\
\text { Hermenéutica, } \\
\text { interacción, } \\
\text { creatividad }\end{array}$ & $\begin{array}{l}\text { Religión, } \\
\text { Normas, } \\
\text { Emblemas, } \\
\text { Ejemplos, } \\
\text { Protocolos, } \\
\text { celebraciones, } \\
\text { tendencias, modas, } \\
\text { juegos, } \\
\text { Modos de vida }\end{array}$ & $\begin{array}{l}\text { Alimentos, utensilios, } \\
\text { vestuario, vivienda, } \\
\text { decoración }\end{array}$ & $\begin{array}{l}\text { Información, medir } \\
\text { tiempo, } \\
\text { construir, iluminar, } \\
\text { acueducto, , procesar } \\
\text { y alargar vida } \\
\text { alimentos, purificar, } \\
\text { obtener energía, } \\
\text { transportar, proyecter } \\
\text { imágenes, prótesis, } \\
\text { vestuario, } \\
\text { salud, seguridad }\end{array}$ & $\begin{array}{l}\text { Sociedad, } \\
\text { economía, política, } \\
\text { religión, arte, } \\
\text { educación, historia, } \\
\text { literatura, } \\
\text { geografía, ciencia } \\
\text { natural } \\
\text { matemática, } \\
\text { química, física, } \\
\text { leyes, milicia }\end{array}$ & $\begin{array}{l}\text { Imperios, } \\
\text { Lideres, } \\
\text { Hegemonia, } \\
\text { guerra, paz, tirania, } \\
\text { dominación, } \\
\text { colonización, } \\
\text { esclavitud, } \\
\text { independencia, } \\
\text { comunidad, } \\
\text { dictadura, } \\
\text { monarquia, } \\
\text { democracia, } \\
\text { socialismo, } \\
\text { Globalización, } \\
\text { Humanidad, } \\
\text { Cooperación, } \\
\text { Participación } \\
\text { ciudadana }\end{array}$ \\
\hline $\begin{array}{l}\text { Texto, } \\
\text { Cultura de registro, } \\
\text { Comunicación }\end{array}$ & $\begin{array}{l}\text { Expresión del ritmo } \\
\text { universal, } \\
\text { Tradición, } \\
\text { Turismo }\end{array}$ & $\begin{array}{l}\text { Productividad, } \\
\text { Hábitats, Cultura } \\
\text { ambientaly } \\
\text { Sostenibilidad }\end{array}$ & $\begin{array}{l}\text { Calidad de vida, lujo, } \\
\text { Desarrollo, } \\
\text { Investigación, } \\
\text { Patrimonio }\end{array}$ & $\begin{array}{l}\text { Epocas, } \\
\text { Ciencia, } \\
\text { Disciplinas, Arte, } \\
\text { Educación }\end{array}$ & $\begin{array}{l}\text { Euforia, Reto } \\
\text { manipulación, } \\
\text { masificación, } \\
\text { cosificación, } \\
\text { defensa }\end{array}$ \\
\hline
\end{tabular}

Fuente: Elaboración propia.

3.2 Comprobación. Se basa en la experimentación en la que se atiende a la formulación de hipótesis, la inducción, la deducción, la demostración y la justificación.

3.3 Estudios cronológicos. En los que se hace necesaria la prueba de carbono y la de potasio radioactivo según date la evidencia del hecho cultural.

4.4 Intervención cultural. A través de la propuesta, aceptación, participación y desarrollo de programas en mira al mejoramiento comunitario, local, regional y aún nacional, es posible generar culturas específicas como la cultura ciudadana, de la sostenibilidad ambiental, de la productividad, de la paz, de la afectividad, de la calidad académica, de la calidad de vida, de la salubridad, de la
3.5 La investigación heurística de carácter cualitativo es una forma de indagación o investigación para lograr un reconocimiento puntual del objeto de estudio apoyado en materiales categoriales. Se puede observar en la tabla 1 una herramienta cognitiva para precisar algunos alcances heurísticos sobre la especificidad de la Cultura: 


\section{DISCUCIÓN Y ANÁLISIS DE RESULTADOS:}

Del estudio heurístico realizado, es posible extraer variables e indicadores que permitan canalizar estudios etnográficos 0 indagativos de lo cultural. Un ejemplo de ello, puede evidenciarse en la cultura ambiental como nueva región de conocimiento en la que se pueden precisar las siguientes variables de observación y posible intervención:

Imaginario cultural sobre el recurso natural y el medio ambiente: corresponde a cada noción, idea, concepto y aún teoría que se tiene sobre el origen del recurso natural, sus características, clases, su renovación, entre otras.

Evidencia de conciencia sobre el recurso natural y el medio ambiente y su vulnerabilidad: No basta contar con un imaginario sobre los recursos naturales, se requiere de la conciencia de cómo se puede contribuir para su cuidado y mantenimiento. Tener la conciencia de que todo repercute en todo.

Hábitos cotidianos que impactan el recurso natural y lo ambiental: Realizar inventario de cada uno de los hábitos cotidianos que pueden impactar en los recursos naturales y el medio ambiente.

Mecanismos y estrategias para propiciar la conciencia ambiental reflexiva: alude a aquello que posibilita el pensamiento metacognitivo permanente y el análisis de situaciones o casos específicos bajo la técnica visual, de expresión grupal o de indagación documental.

Estrategias de desentronización de malos hábitos ambientales: Tiene que ver con la sustitución gradual de prácticas que afectan el medio ambiente.

Estrategias de entronización de buenos hábitos ambientales: Tiene que ver con la implantación gradual de prácticas que favorezcan el medio ambiente.

Mecanismos para la entronización de la tradición ambiental: Tiene que ver con la implantación gradual de prácticas que permitan instaurar una tradición ambiental favorable para el medio ambiente.

Inventario de necesidades para la elaboración de códigos científicos-disciplinares ambientales: Tener relacionadas de manera organizada las necesidades ambientales posibilita la priorización de actividades que logren mayor efectividad en materia ambiental.
Temas del estado del arte en materia ambiental: Tópicos de interés inventariados.

Normas para la regulación en uso racional de los recursos naturales. Registro.

Mecanismos de control en el uso racional de los recursos naturales. Coherente con las normas. Observación de los mismos en situaciones específicas.

Listado de acontecimientos ambientales propiciables: permite analizar la situación en prospectiva, hallar soluciones ambientales de manera más pertinente $y$ efectiva.

Porcentaje de conservación natural: Se estima a la par de los inventarios realizados sobre biodiversidad.

Indicadores de sostenibilidad ambiental: Se estiman en el análisis de situaciones según el estado del arte sobre sostenibilidad ambiental.

Nivel de salubridad: se verifican según el estado del arte para estudio de situaciones y según el inventario de hallazgos respecto a la salubridad.

Inventario de Biodiversidad.

Número y tema estudios de impacto ambiental. Contribuyen a la conformación del estado del arte en la disciplina.

Cuantificación de los recursos naturales: Requiere de estudios puntuales que genere puntos de referencia a lo largo del tiempo.

Modelación de comportamiento de sistemas ambientales: permite simular condiciones favorables o desfavorables que lleve a la toma de decisiones.

Registros de historia ambiental.

Temas de la política ambiental.

Grado de contaminación. Requiere de estudios puntuales.

Escala de riesgo. Requiere de estudios puntuales.

Alertas de Amenaza. Requiere de estudios puntuales.

Planificación en el ordenamiento territorial. Requiere de estudios puntuales.

Componentes de la gestión integral ambiental. 
Doris Vanegas Vanegas - Jacipt Alexander Ramón Valencia - Jarol Derley Valencia

Espacios de participación ciudadana en materia ambiental.

Preguntas en relación a lo ambiental.

Listado de problemas en relación a lo ambiental.

Protocolos de registro en manejo ambiental.

Este desglose de algunas variables, permite tener un punto de referencia sobre lo que se requiere resolver en una cultura ambiental, considerada nueva región de conocimiento con todo y su complejidad; y, necesitada de la intervención de todos, ya que el reto para entronizarla cabalmente es socio-cultural.

Dentro de los resultados significativos de este estudio, tenemos la aclaración de unos principios guía o referente, la representación cognitiva del constructo cultural en su contextualización; una síntesis de las diversas concepciones de la Cultura a lo largo del tiempo; la sinopsis de la diversidad de componentes y rasgos culturales; las categorías y macroestructuras planteadas como características y clasificación de la cultura; los criterios, los métodos de investigación e intervención; y, el desglose de variables de observación y posible intervención para el caso de la cultura ambiental como nueva región de conocimiento. Aspectos que no habían sido puntualizados desde una mirada heurística.

\section{CONCLUSIONES:}

Se han logrado definir los aspectos relevantes de la Cultura, sus conceptos inmediatos, su estudio a lo largo del tiempo, sus categorías y una sencilla pero puntual presentación de su productividad.

Se ha realizado un abordaje de la Cultura de manera heurística demostrando la utilidad del método elegido.

Se precisaron principios, elementos, criterios, categorías métodos y variables, a manera de prolegómenos, para dar a conocer el estado del arte cultural con el que contamos hasta el momento.

Este estado del arte se diferencia de otros en la medida en que puntualiza particularidades de la Cultura que no han sido abordadas globalmente.

La concepción de la Cultura que más se ajusta a las necesidades de formación de una cultura ambiental es la que estima que la Cultura "se transforma en el recurso fundamental para el desarrollo de comunidad y sociedades creativas, que permite la socialización del saber y el cultivo de las actitudes solidarias necesarias para alcanzar el desarrollo sostenible". (Barbero, 2011). (Mirada de la sostenibilidad ambiental y el desarrollo local a partir de la intervención cultural).

\section{REFERENCIAS:}

Bajtin, M. (2011). Las fronteras del discurso. El problema de los géneros discursivos. El hablante en la novela. Buenos Aires: Las cuarenta.

Barbero, A. M. (2011). La gestión del patrimonio histórico como instrumento para un desarrollo sostenible. Un caso práctico: El proyecto de desarrollo local "os ambientes do ar". Salamanca: Vítor.

Carvajal, J. A. (1996). Semiología de Códigos Culturales. Pamplona, Colombia: Universidad de Pamplona.

Geertz, C. (1987). La interpretación de las culturas. Barcelona: Gedisa.

Hall, S. (1981). La cultura, los medios de comunicación y el efecto ideológico en Sociedad y Comunicación de Masas de Curran, J. et al., Fondo de Cultura Económica, México.

Halliday, M. A. K. (2013). El lenguaje como semiótica social. México, México: Fondo de Cultura Económica.

Lévi-Strauss, C. (1977). Antropología estructural. Buenos Aires: Eudeba [1953].

López, A. y Vanegas, D. (2005). El conocimiento del texto lingüístico y su trascendencia en la Didáctica de la Lengua y la Literatura. Bordón. Vol. 57 No. 3 p. 397-405.

López, A., Vanegas, D. y López, M. (2007). Una competencia Texto-lingüística en permanente desarrollo. El Guiniguada. Vol15-16, p. 31 - 42.

Magendzo, A. (2008). Dilema del currículum y la pedagogía. Santiago: LOM Ediciones.

Martínez, M.A. (1999). El enfoque sociocultural en el estudio del desarrollo y la educación. Ciudad de México, México: Universidad Nacional Autónoma de México. 
ISSN: 1794-9920 Julio - Diciembre 2015

Marx, K. (1965). Ideología alemana. México: Ediciones de Cultura Popular.

Morin, E. (1994). Introducción al pensamiento complejo. Barcelona: Gedisa.

Mosterín, J. (1993). Filosofía de la cultura. Alianza Editorial, Madrid.

Steward, J. (1992). "El concepto y el método de la ecología cultural". En: Bohannan, P y Glazer, M. (comp.) ().: Antropología. Lecturas. Madrid: McGraw-Hill.

Torres, J. (1996). El Currículum oculto. Madrid: Morata.

Vanegas, D. y López, A. (2005). Experiencia didáctica en el desarrollo de la competencia textolingüística como orientadora de la lectura y la escritura (estudio experimental). Diexpe Experimenta Pedagógicamente. Vol.5, p.15 - 24

Vigotsky, L. S. (1973). Pensamiento y lenguaje: teoría del desarrollo cultural de las funciones psíquicas. Buenos Aires: La Pléyade (1934).

White, L. A. (1992). "La energía y la evolución de la cultura". En: Bohannan, P y Glazer, M. (comp): Antropología. Lecturas. McGraw-Hill. Madrid. 